\title{
Allergen immunotherapy
}

\author{
Sonia Mannan*,1 \\ ${ }^{1}$ Future Science Group, Unitec House, 2 Albert Place, London, N3 1QB, UK \\ * Author for correspondence: Tel.: +44 208371 6090; s.mannan@futuremedicine.com
}

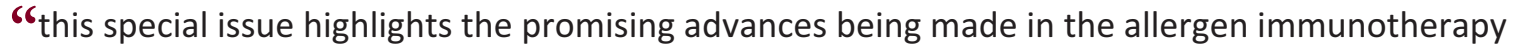 field, challenges that need to be overcome to ensure continuing progress, as well as future directions."

First draft submitted: 02 November 2017; Accepted for publication: 02 November 2017; Published online: 14 November 2017

We are delighted to announce the release of our latest themed issue, which highlights developments in the field of allergen immunotherapy.

During the last few decades, there has been an increase in the prevalence of allergies and it has been predicted that by 2025, half of the entire EU population will be affected [1]. Allergen-specific immunotherapy is currently being explored as a potential preventive treatment for allergic reactions elicited by substances such as pollen, house dust mites and peanuts [2]. The aim of immunotherapy has been to direct the immune response towards cellular immunity and decrease the production of specific IgE antibodies, thus reducing the symptoms of allergy for the sufferers [3].

This special focus issue covers the latest advances in allergen immunotherapy covering both environmental and food allergies, with the aim of directing the improvement and optimization of immunotherapy for allergy specific treatment.

Land and Chen begin the issue with their Editorial discussing whether consuming baked products containing milk and eggs could be used as an effective alternative to oral immunotherapy in individuals with milk and egg allergies [4]. In a Preliminary Communication, Gamazo et al. develop a model of peanut-induced anaphylaxis to study immunotherapy approaches and examine the effect of immunogenic peanut extract encapsulated polymeric nanoparticles on anaphylaxis and immunological outcomes [5]. Ciprandi et al. investigated evaluate the relationships between climate and house dust mite allergic sensitization in Italy in their Short Communication article [6]. We have several Original Research Articles in this issue, the first from Mösges et al. where the effect of different doses of carbamylated monomeric allergoid tablets in patients with grass allergy is analysed [7]. Chaudhuri et al. present data indicating that allergen immunotherapy decreases the sensitivity to apoptosis of Treg cells via extrinsic, intrinsic and perforin/granzyme pathways [8]. Schulze et al. examine the predictive value of in vitro IgE tests in children and adolescents with allergic asthma and sensitization to house dust mites [9]. Bahceciler et al. examine the effects of sublingual immunotherapy in asthmatic children [10]. In their Special Report, Incorvaia et al. explore allergen-specific immunotherapy for birch-apple syndrome sufferers [11]. Molnár et al. summarize ragweed specific sublingual immunotherapy strategies and challenges in their 3-year study [12]. Finally concluding the issue is a Review by Pisi et al. evaluating the role of microbiome in shaping the immune system as a possible causative mechanism leading to asthma and potential therapeutic approaches [12].

In summary, this special issue highlights the promising advances being made in the allergen immunotherapy field, challenges that need to be overcome to ensure continuing progress, as well as future directions. We hope that the contents of this issue will provide a valuable resource for the research community and that the expert perspectives presented will help drive this exciting area forward. 


\section{References}

1 (EAACI, 2016). www.eaaci.org/documents/EAACI_Advocacy_Manifesto.pdf

2 American College of Allergy, Asthma \& Immunology. http://acaai.org/allergies/allergy-treatment/allergy-immunotherapy

3 Tan T, Ellis JA, Saffery R, Allen KJ. The role of genetics and environment in the rise of childhood food allergy. Clinical and Experimental Allergy (42) 20-29 (2012).

4 M Chen, M Land. Baked Milk and Baked Egg Oral Immunotherapy. Immunotherapy 9(15), 1201-1204 (2017).

5 Gamazo C, García-Azpíroz M, Rebouças JDe S et al. Oral immunotherapy using polymeric nanoparticles loaded with peanut proteins in a murine model of fatal anaphylaxis. Immunotherapy 9(15), 1205-1217 (2017).

6 Ciprandi G, Puccinelli P, Incorvaia C, Passalacqua G. The relevance of House Dust Mites allergy in clinical practice: the epidemiological impact on allergen immunotherapy. Immunotherapy 9(15), 1219-1224 (2017).

7 Mösges R, Christina R, Eichel A et al. Dose-finding study of carbamylated monomeric allergoid tablets in grass-allergic rhinoconjunctivitis patients. Immunotherapy 9(15), 1225-1238 (2017).

8 Datta A, Moitra S, Das PK et al. Allergen Immunotherapy modulates sensitivity of regulatory T cells to apoptosis in a rat model of allergic asthma. Immunotherapy 9(15), 1239-1251 (2017).

9 Schulze J, Leberkuehne L, Salzmann-Manrique E, Schubert R, Zielen S, Rosewich M. Comparison of two different assays and the predictive value of allergen components in house dust mite allergy. Immunotherapy 9(15), 1253-1262 (2017).

10 Bahceciler NN, Galip N, Babayigit A. Steroid sparing effect of sublingual immunotherapy: real life study in mono/polisensitized children with asthma. Immunotherapy 9(15), 1263-1269 (2017).

11 Incorvaia C, Ridolo E, Mauro M, Russello M, Pastorello E. Allergen Immunotherapy for birch-apple syndrome: what do we know? Immunotherapy 9(15), 1271-1278 (2017).

12 Molnár V, Nagy A, Tamási L et al. From genomes to diaries: a 3-year prospective, real-life study of ragweed specific sublingual immunotherapy. Immunotherapy 9(15), 1279-1294 (2017).

13 Pisi G, Fainardi V, Aiello M, Bertorelli G, Crisafulli E, Chetta A. The role of microbiome in childhood asthma. Immunotherapy 9(15), 1295x-1304 (2017). 